Document downloaded from:

http://hdl.handle.net/10251/139466

This paper must be cited as:

Tur Valiente, M.; Baeza González, LM.; Fuenmayor Fernández, F.; Garcia, E. (2014). PACDIN statement of methods. Vehicle System Dynamics. 53(3):402-411. https://doi.org/10.1080/00423114.2014.963126

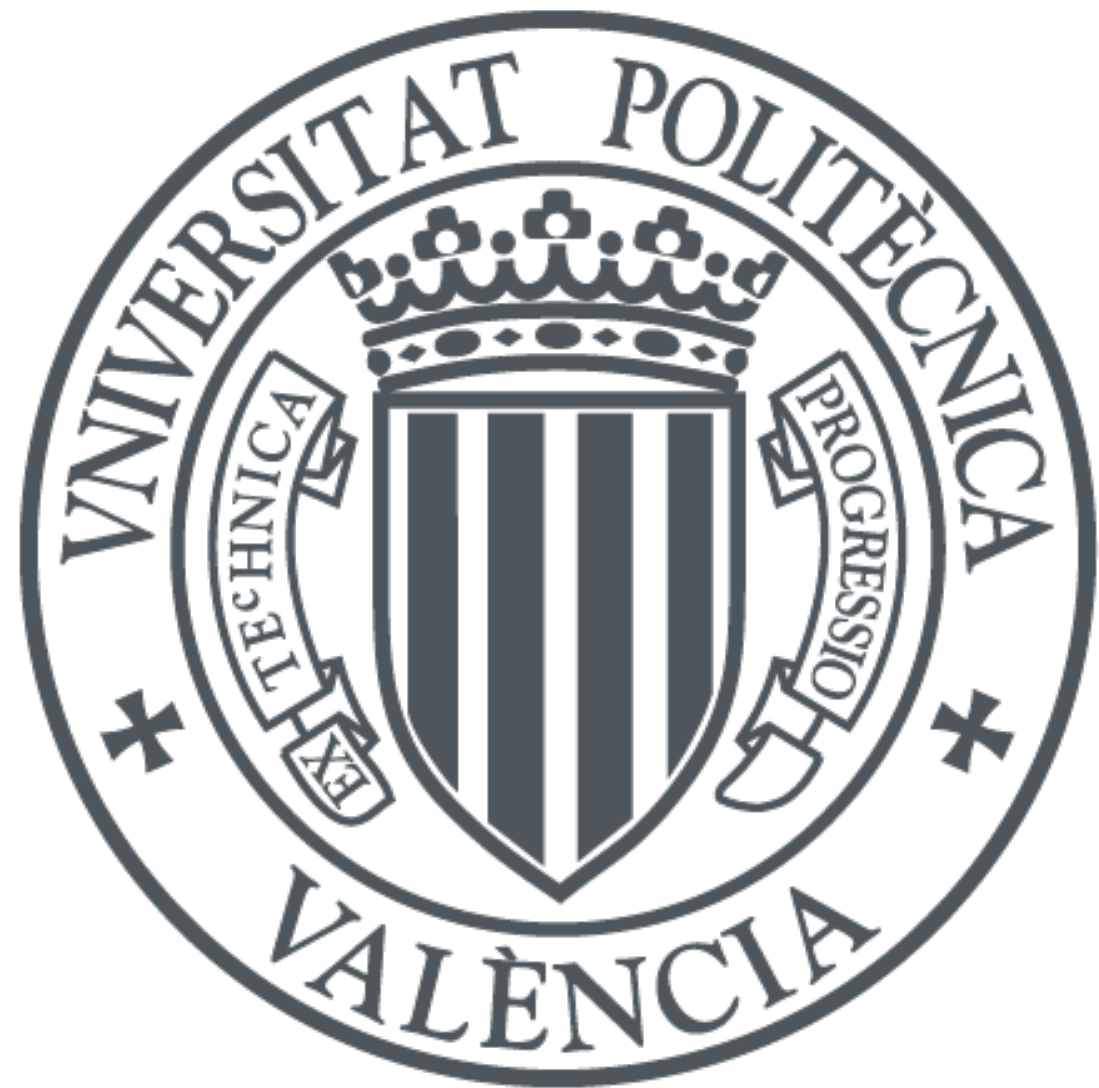

The final publication is available at

https://doi.org/10.1080/00423114.2014.963126

Copyright Taylor \& Francis

Additional Information

This is an Accepted Manuscript of an article published by Taylor \& Francis in Vehicle System Dynamics on 2014 available online: https://doi.org/10.1080/00423114.2014.963126 


\title{
PACDIN Statement of Methods
}

\author{
M. Tur $^{a *}$, L. Baeza ${ }^{a}$, F.J. Fuenmayor ${ }^{a}$ and E. García ${ }^{b}$ \\ ${ }^{a}$ Centro de Investigación de Tecnología de Vehículos, Universitat Politècnica de València, \\ Camino de Vera s/n, 46022 Valencia (Spain); b Patentes Talgo S.L., Paseo del Tren
}

Talgo 3, 28290 Las Matas (Spain)

\begin{abstract}
PACDIN (PAntograph Catenary Dynamic Interaction) is a code developed by the vehicle technology research centre (CITV) of the Universitat Politècnica de València in collaboration with the railway company Talgo S.L. The model of the catenary is a finite element model using absolute nodal coordinates. It is based on a general formulation that can be applied to analysing a wide range of catenary configurations, including stitch wire, transitions or non-straight path tracks. The formulation is fully non-linear and includes large deformations, dropper slackening and contact interaction. The model is linearized when deformations are small, as in the case of the benchmark dynamic analysis. The results of the PACDIN code show a good agreement with the average results of other benchmark codes.
\end{abstract}

Keywords: catenary; dynamics; pantograph; benchmark; absolute nodal coordinate

\section{Introduction}

The PACDIN code (PAntograph-Catenary Dynamic INteraction) was developed by the vehicle technology research center (CITV) of the Universitat Politècnica de València in collaboration with the railway company Talgo S.L., in the context of a project set in motion in 2010. The code is based on the finite element method and uses 3D absolute nodal coordinate formulation (ANCF) wire elements, nonlinear bars and spring and mass elements to model catenary components. The pantograph is simulated as a lumped mass model. Different configurations of the overhead equipment are supported i.e. catenary with or without stitch wire, curved tracks, etc. The code is fully developed in Matlab ${ }^{\circledR}$ and an interactive graphical user interface (GUI) allows the user to choose different simulation options.

The core of the code is divided into 4 independent modules:

- Data input and mesh generation: Using a general geometric description of the catenary (number of supports, number of droppers and inter-dropper distance, height of contact wire, etc.) and the desired number of finite elements, the topology of the mesh is automatically defined and an estimate is made of the coordinates of each node and undeformed element lengths.

- Initial configuration: Based on the abovementioned mesh, a nonlinear problem is solved to find the coordinates of each node and the undeformed element length that allows the constraints imposed during the overhead assembly to be fulfilled, i.e. tension in messenger, contact and stitch wires, position of the droppers along the track path, height of the contact wire in connection with droppers, etc.

\footnotetext{
*Corresponding author. Email: manuel.tur@mcm.upv.es
} 


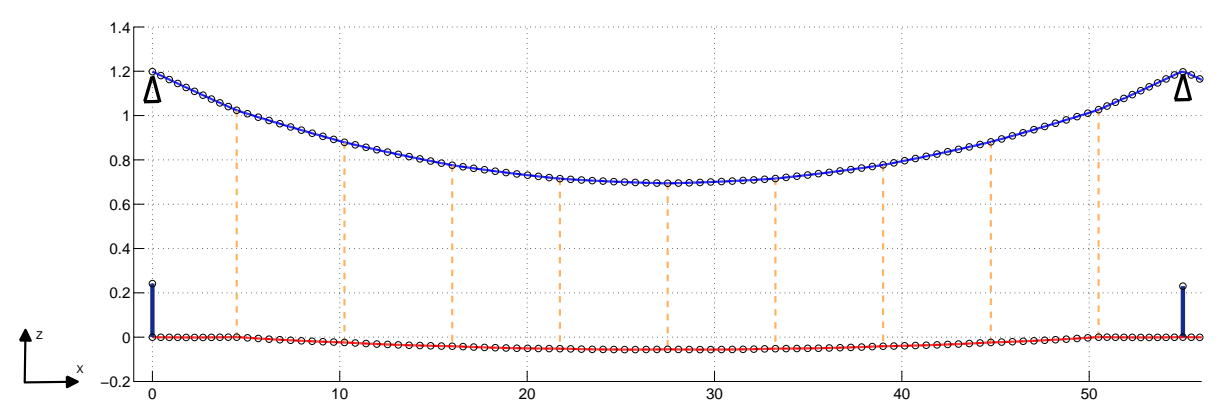

Figure 1. Finite element mesh. Continuous line: ANCF elements, dashed line: bar elements with slackening, thick line: bar elements, triangle symbol: spring-damper elements.

- Dynamic analysis: Performs the time integration of the equations using the parameters defined by the user. Three methods are available, the Hughes-HilbertTaylor (HHT), Newmark, and 4th order Runge-Kutta, all with a linearized or complete non-linear model of the system.

- Postprocessor: An interactive Matlab GUI allows the user to plot the results in graphs (contact force, pantograph position, etc.), to compute the statistics of the simulation, etc.

\section{Methods as applied in the benchmark}

\subsection{Catenary Model}

As pointed out above, the catenary is modelled using the finite element method. A non-linear large deformation behaviour is assumed to develop the system of equations and solve the initial configuration problem, although the model can be linearized to perform time integration. All the nodes of the mesh have 6 degrees of freedom, the three components of the global position of the node and three slopes that are used to define the direction of ANCF elements.

Twenty identical spans were used for the simulations with a single pantograph and 30 spans for the simulations with two pantographs. The finite element mesh of a single span is shown in figure 1 where the circles correspond to the nodes of the mesh. The global axes are also shown. The $x$ axis is aligned with the direction of the track and $z$ axis is the vertical direction. There are 10 elements between droppers and between steady arm and droppers in the contact and messenger wires, for which ANCF elements are used. Steady arms and droppers are modelled as single elements. Non-linear bar elements are used for the former and bar elements with slackening for the latter. The steady arm fixed point $y$ coordinate is defined to obtain a stagger of the contact wire and coordinate $z$ to achieve the prescribed height $(z=0)$ of the contact wire in the connexion with the steady arm.

In what follows we briefly summarize the main features of the element forces computation and the formulation using global coordinates.

\subsubsection{Contact and messenger wires}

The contact and messenger wires are defined using ANCF elements [1-3]. In figure 2 a) we show an element with nodes $i$ and $j$. In this case, the element degree of freedom vector $\mathbf{q}_{\mathrm{c}}$ has 12 components, 6 for each node. In this formulation, no infinitesimal or finite rotations are used as nodal coordinates. Instead, the global displacement coordinates and slopes are used as defined in equation 1. Variable $\chi \in$ $\left[0, l_{0}\right]$ denotes the position of an arbitrary point of the element in the undeformed 


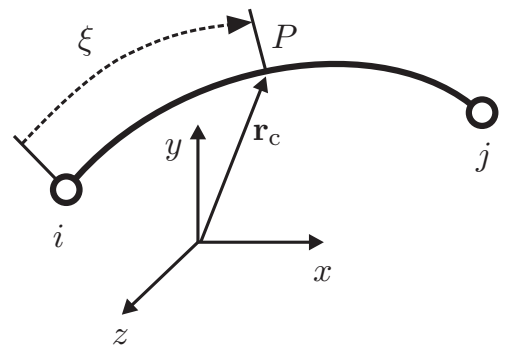

(a)

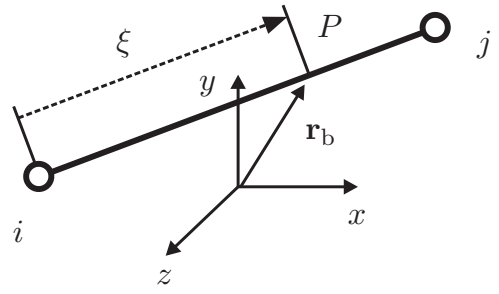

(b)

Figure 2. Global position of the two elements used. Left: The configuration of the ANCF element is a cubic polynomial. Right: The bar element is a linear polynomial, i.e. a straight line.

reference configuration, where $l_{0}$ is the undeformed length.

The global configuration of this element is assumed to be a cubic polynomial whose coefficients are computed by imposing the continuity of the position and the slopes in each node. The element therefore has a smooth deformed configuration with $C^{1}$ continuity. The interpolation that defines the global position at an arbitrary point $P$ can be computed as a function of the normalized local coordinate of the point $\xi=\chi / l_{0} \in[0,1]$ and the undeformed length $l_{0}$ as:

$$
\begin{aligned}
& \mathbf{r}_{\mathrm{c}}=\mathbf{S}_{\mathrm{c}} \mathbf{q}_{\mathrm{c}} \quad \text { where } \quad \mathbf{q}_{\mathrm{c}}=\left[x_{i} y_{i} z_{i} \frac{\partial x_{i}}{\partial \chi} \frac{\partial y_{i}}{\partial \chi} \frac{\partial z_{i}}{\partial \chi} x_{j} y_{j} z_{j} \frac{\partial x_{j}}{\partial \chi} \frac{\partial y_{j}}{\partial \chi} \frac{\partial z_{j}}{\partial \chi}\right]^{\mathrm{T}} \\
& \mathbf{S}_{\mathrm{c}}=\left[\begin{array}{cccccccccccc}
S_{1} & 0 & 0 & S_{2} & 0 & 0 & S_{3} & 0 & 0 & S_{4} & 0 & 0 \\
0 & S_{1} & 0 & 0 & S_{2} & 0 & 0 & S_{3} & 0 & 0 & S_{4} & 0 \\
0 & 0 & S_{1} & 0 & 0 & S_{2} & 0 & 0 & S_{3} & 0 & 0 & S_{4}
\end{array}\right] \\
& S_{1}(\xi)=1-3 \xi^{2}+2 \xi^{3} \\
& S_{3}(\xi)=3 \xi^{2}-2 \xi^{3}(\xi)=l_{0}\left(\xi-2 \xi^{2}+\xi^{3}\right)
\end{aligned}
$$

The ANCF element can transmit forces due to axial and bending deformation. The elastic forces of this element $\mathbf{f}_{\mathrm{c}}^{\mathrm{e}}$ can be computed $[1,3]$ taking the derivative of the strain energy with respect to the degrees of freedom as

$$
\mathbf{f}_{\mathrm{c}}^{\mathrm{e}}\left(\mathbf{q}_{\mathrm{c}}, l_{0}\right)=\frac{\partial U_{\mathrm{c}}}{\partial \mathbf{q}_{\mathrm{c}}}, \quad U_{\mathrm{c}}=\frac{1}{2} \int_{0}^{1}\left(E A \varepsilon_{\mathrm{L}}^{2}+E I \kappa^{2}\right) l_{0} d \xi
$$

where

$$
\begin{aligned}
& \varepsilon_{\mathrm{L}}=\frac{1}{2}\left(\mathbf{r}_{\mathrm{c}}^{\prime \mathrm{T}} \mathbf{r}_{\mathrm{c}}{ }^{\prime}-1\right)=\frac{1}{2}\left(\mathbf{q}_{\mathrm{c}}{ }^{\mathrm{T}} \mathbf{S}_{\mathrm{c}}^{\prime \mathrm{T}} \mathbf{S}_{\mathrm{c}}^{\prime} \mathbf{q}_{\mathrm{c}}-1\right) \\
& \kappa=\left|\mathbf{r}_{\mathrm{c}}{ }^{\prime \prime}\right|=\sqrt{\mathbf{q}_{\mathrm{c}}{ }^{\mathrm{T}} \mathbf{S}_{\mathrm{c}}{ }^{\prime \prime}{ }^{\mathrm{T}} \mathbf{S}_{\mathrm{c}}{ }^{\prime \prime} \mathbf{q}_{\mathrm{c}}}
\end{aligned}
$$

In the last equation $E$ is the Young's modulus, $A$ is the area and $I$ is the moment of inertia, $\varepsilon_{\mathrm{L}}$ is the axial deformation and $\kappa$ the curvature. The prime symbol ' is used to denote the derivative with respect to the local coordinate $\chi$. Note that the strain energy includes two terms due to axial and bending deformation. The value of the curvature is a valid approximation when axial deformation is small enough, as in the case of catenary wires. The ANCF element can be improved [4] to take 
into account discontinuity in the axial stress, which is caused when a catenary wire is connected to another element such as a dropper or a steady arm.

The mass matrix is computed from the expression of the kinetic energy of the element as:

$$
T_{\mathrm{c}}\left(\mathbf{q}_{\mathrm{c}}, l_{0}\right)=\frac{1}{2} \int_{0}^{1} \rho \dot{\mathbf{r}}_{\mathrm{c}}^{\mathrm{T}} \dot{\mathbf{r}}_{\mathrm{c}} l_{0} d \xi=\frac{1}{2} \dot{\mathbf{q}}_{\mathrm{c}}^{\mathrm{T}}\left(\int_{0}^{1} \rho \mathbf{S}_{\mathrm{c}}^{\mathrm{T}} \mathbf{S}_{\mathrm{c}} l_{0} d \xi\right) \dot{\mathbf{q}}_{\mathrm{c}}=\frac{1}{2} \dot{\mathbf{q}}_{\mathrm{c}}^{\mathrm{T}} \mathbf{M}_{\mathrm{c}} \dot{\mathbf{q}}_{\mathrm{c}}
$$

where $\rho$ is the density per unit length, $\dot{\mathbf{r}}$ is the time derivative of the position vector and $\dot{\mathbf{q}}_{\mathrm{c}}$ is the time derivative of the vector of nodal coordinates.

\subsubsection{Steady arms}

Steady arms are simulated using non-linear bar elements. As shown in figure 2b) this element has only two nodes and its configuration is a straight line. The element vector of degrees of freedom of this element $\mathbf{q}_{\mathrm{b}}$ has only six components, the global position of both nodes. It can only transmit forces in the axial direction and can undergo large rotations without transmitting forces. The elastic force vector for a given element $\mathbf{f}_{\mathrm{b}}^{\mathrm{e}}$ can be computed as a function of the global coordinates of the $i$ ans $j$ nodes and the undeformed length $l_{0}$ as

$$
\mathbf{f}_{\mathrm{b}}^{\mathrm{e}}\left(\mathbf{q}_{\mathrm{b}}, l_{0}\right)=\frac{\partial U_{\mathrm{b}}}{\partial \mathbf{q}_{\mathrm{b}}} \quad U_{\mathrm{b}}=\frac{1}{2} \int_{0}^{1} E A \varepsilon_{\mathrm{L}}^{2} l_{0} d \xi
$$

where

$$
\varepsilon_{\mathrm{L}}=\frac{l_{d}^{2}-l_{0}^{2}}{2 l_{0}^{2}} \quad \text { and } \quad l_{d}^{2}=\left(x_{j}-x_{i}\right)^{2}+\left(y_{j}-y_{i}\right)^{2}+\left(z_{j}-z_{i}\right)^{2}
$$

The mass matrix for the bar element is computed using equation (3) but replacing the shape functions of the ANCF element $\mathbf{S}_{\mathrm{c}}$ by that of the bar element $\mathbf{S}_{\mathrm{b}}$ which is defined as

$$
\mathbf{S}_{\mathrm{b}}=\left[\begin{array}{cccccc}
S_{1} & 0 & 0 & S_{2} & 0 & 0 \\
0 & S_{1} & 0 & 0 & S_{2} & 0 \\
0 & 0 & S_{1} & 0 & 0 & S_{2}
\end{array}\right] \quad S_{1}(\xi)=1-\xi \quad S_{2}(\xi)=\xi
$$

In the benchmark data the steady arm is defined as a deformable bar with a given non-straight geometry. Bending and axial deformations are allowed. In our simulation we computed an equivalent straight bar having the same stiffness in the axial direction and an equivalent mass. For this purpose a finite element model of the steady arm was used. As shown in figure 3, the steady arm model was constrained in such a way that only axial displacement was allowed. The ratio between the applied force and the axial displacement defines the stiffness of the straight bar used in our model, i.e. $E A=79280.07 \mathrm{~N}$. The length of the straight bar is computed as $l_{0}=\sqrt{1.2^{2}+0.2^{2}}=1.216 \mathrm{~m}$. The mass per unit length is accordingly modified to preserve the total mass, so $\rho=0.7727 \mathrm{~kg} / \mathrm{m}$.

Although PACDIN code has a 3D formulation, it can also be used to construct a 2D catenary model. Catenary stagger is set to zero and the displacement in the $y$-direction is constrained in all nodes. The steady arm is approximated as a vertical bar with a stiffness parameter chosen to have the same vertical stiffness as in the 3D case. The density is also modified to have an equivalent mass. In this case, the length of the bar is chosen arbitrarily to be $l_{0}=1 \mathrm{~m}$ so the parameter $E A=274.07 \mathrm{~N}$ and the mass per unit length $\rho=0.94 \mathrm{~kg} / \mathrm{m}$. 


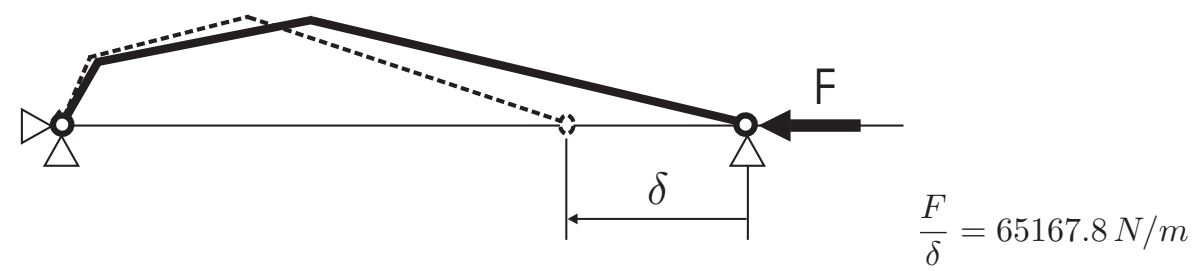

Figure 3. Axial stiffness of the steady arm defined in the Benchmark.

\subsubsection{Droppers and clamps}

The same formulation of the bar element presented above is used to model the droppers, except that in this case it is not allowed to transmit compressive forces. Therefore, if the axial deformation is positive, the elastic force is computed using equation (4). If the axial deformation is negative, the elastic force is zero.

Clamps are introduced as point mass elements located in the nodes of the droppers, using the value prescribed in the benchmark data.

\subsubsection{Spring-damper elements}

As pointed out in the benchmark data definition, the supports of the messenger wire are simulated by a suspension having a spring in parallel to a damper. The elastic force of this element is acting in the vertical direction. It can be computed as:

$$
\mathbf{f}_{\mathrm{sd}}^{\mathrm{e}}(\mathbf{q})=\left\{\begin{array}{lll}
0 & 0 & k_{w}\left(z-z_{\mathrm{sd}}\right)+c_{w} \dot{z}
\end{array}\right\}^{T}
$$

where $k_{w}$ is the stiffness, $c_{w}$ is the damping and $z$ is the global coordinate of the node in the messenger wire connected to the suspension. $z_{s d}$ is the reference position of the support where the transmitted force is equal to zero. This value is computed in the solution of the static configuration of the system together with the undeformed length and the steady arm height to fulfil the geometric definition of the catenary given in the benchmark.

\subsection{Pantograph model}

The pantograph defined in the benchmark has three degrees of freedom. In the formulation these degrees of freedom are the global coordinates of every mass (see Figure 4). In the PACDIN code the force exerted by the pantograph on the catenary depends on two external parameters that must be defined by the user, as depicted in Figure 4 . The two parameters are the external pantograph force $F_{p}$ and the reference position $z_{p}$ of the pantograph base.

The benchmark data imposes a given value of the mean contact force on the 10 central spans of the simulation for every train speed, so there are many combinations of the two parameters that can be defined to achieve this constraint. In the simulations we computed $z_{p}$ as the $z$ coordinate of the contact wire in the initial position of the pantograph, i.e. in the first steady arm. Therefore, the reference position is $z_{p}=0 \mathrm{~m}$ as this is a prescribed value in the definition of the catenary. The value $F_{p}$ is obtained for every simulation in an iterative process, to achieve the prescribed value of the contact mean force. In Table 1 the values of forces are shown for every simulation case and speed. As the pantograph stiffness $k_{1}$ is very small, $z_{p}$ has a small influence in the dynamic interaction results. Using this methodology, the mean value defined in the Benchmark data is fulfilled with $\pm 0.1 \mathrm{~N}$ of precision.

In simulation Case 3 (dynamic with two pantographs) the trailing pantograph is not in contact with the catenary at the beginning of the simulation. The same 
Table 1. Pantograph force $F_{p}$ used in the simulations (Newtons).

\begin{tabular}{|l|c|c|c|c|c|}
\hline Speed $(\mathrm{km} / \mathrm{h})$ & 10 & 230 & 275 & 320 & 365 \\
\hline case 2a & $103.58 / 212.35$ & 127.56 & 152.25 & 182.17 & 185.28 \\
\hline case 2b & $103.57 / 212.33$ & 127.52 & 152.26 & 182.00 & 184.00 \\
\hline case 3 (leading) & - & 127.63 & 152.23 & 182.12 & 185.31 \\
case 3 (trailing) & - & 127.61 & 151.29 & 183.06 & 187.41 \\
\hline
\end{tabular}

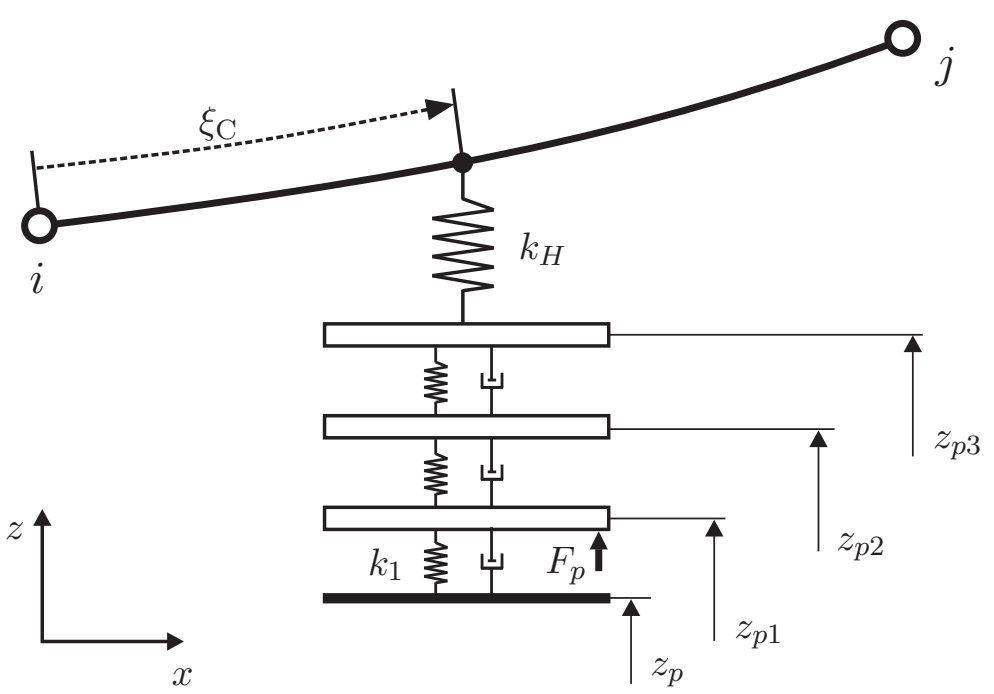

Figure 4. Model of the pantograph-catenary contact.

value of $z_{p}$ is used for the trailing pantograph. The values of $F_{p}$ and $z_{p}$ for this pantograph are increased linearly from a lower value (zero force and $z_{p}=-0.02 \mathrm{~m}$ reference position) to the maximum value along a distance of $70 \mathrm{~m}$, beginning when the trailing pantograph reaches the first catenary mast. .

\subsection{Model of pantograph-catenary contact}

The contact between pantograph and catenary is solved using a penalty method [5]. The value of the contact stiffness used in the simulations is $k_{H}=50000 \mathrm{~N} / \mathrm{m}$. The chosen value avoids ill-conditioning of the stiffness matrix while keeping an acceptable accuracy of the results and is used in the bibliography [6].

As depicted in Figure 4, in every time step, the contact point of the pantograph in the catenary must be found. This point is defined using the local coordinate $\xi_{C}$ of the contact point in the corresponding element of the contact wire. The following equation must be solved for every time step to find $\xi_{C}$ :

$$
x_{C}=\mathbf{S}_{\mathrm{c} 1}\left(\xi_{\mathrm{C}}\right) \mathbf{q}_{\mathrm{c}}\left(t_{0}\right)=V \cdot t+s_{0}
$$

where $V$ is the train speed, $t$ the time and $s_{0}$ the initial position of the pantograph. Matrix $\mathbf{S}_{\mathrm{c} 1}$ is the first row of the shape function matrix, defined in equation 2 and $\mathbf{q}_{\mathrm{c}}\left(t_{0}\right)$ is the degree of freedom vector of the particular element in contact. We assume that the displacements are small so we use the position of the catenary elements in the first time step $t_{0}$ to solve this equation.

An interaction element in the form of a spring is added to the model to take the contact into account. This element can only transmit compressive forces in a vertical $z$ direction. The elastic force vector of the interaction element is computed 


$$
\begin{aligned}
& \mathbf{f}_{\mathrm{i}}^{\mathrm{e}}=\left\{\begin{array}{lll}
0 & 0 & F_{c}
\end{array}\right\} \quad \text { with } \\
& F_{c}=k_{H}\left(\mathbf{r}_{\mathrm{c} 3}\left(\xi_{\mathrm{C}}\right)-z_{p 3}\right)=k_{H}\left(\mathbf{S}_{\mathrm{c} 3}\left(\xi_{\mathrm{C}}\right) \mathbf{q}_{\mathrm{c}}-z_{p 3}\right)
\end{aligned}
$$

where $\mathbf{r}_{\mathrm{c} 3}$ is the third component of the vector, i.e. the vertical position of the contact point and $\mathbf{S}_{\mathrm{c} 3}$ is the third row of the shape function matrix (equation 2).

\subsection{Initialisation of the problem (steady state configuration of the catenary)}

As pointed out above, the PACDIN code uses a formulation with global coordinates to define the configuration of each element in the catenary, so that in addition to the global coordinates of the nodes, the undeformed length of the elements is unknown. In [4] we propose a methodology to find the steady state configuration of the catenary and the undeformed length of all the finite elements that would allow the constraints imposed during the catenary stringing to be fulfilled. We applied this methodology to defining the configuration of the catenary of the benchmark and recall here the main features of the computation.

First of all, the catenary must be in equilibrium under the gravitational loads. The elastic force of each element of the mesh is assembled to obtain a global elastic force vector $\mathbf{F}^{\mathrm{e}}$. This includes the ANCF elements (equation 2), the bar elements (equation 4), and the spring elements (equation 6). Similarly the element vector of gravitational loads can be computed and assembled to obtain a global vector $\mathbf{F}^{\mathrm{g}}$. Then the following system of equations can be written

$$
\mathbf{F}^{\mathrm{e}}\left(\mathrm{q}, \mathbf{l}_{0}\right)+\mathbf{F}^{\mathrm{g}}\left(\mathbf{q}, \mathbf{l}_{0}\right)=0
$$

where $\mathbf{q}$ is the vector with all degrees of freedom of the mesh, and $\mathbf{l}_{0}$ the vector containing all the unknown undeformed lengths of the elements.

In addition to this equation, the benchmark data imposes a number of constraints in the wires geometry and tension. The droppers are prescribed to have a given value of the $x$-coordinate, and the dropper-contact wire and steady arm-contact wire connection points have an imposed height value. These restrictions can be written in general as

$$
\mathrm{q}_{i k}=v
$$

where $v$ is the imposed value and the double index in $\mathrm{q}_{i k}$ denotes the degree of freedom $k$ of node $i$.

Instead of using all the length of the elements in the spans as unknowns, we define a design parameter $\beta$ that scales the undeformed length for a group of elements. All the elements of the contact and messenger wires between two droppers or between a steady arm and a dropper belong to a different group. Another design parameter for the span is the vertical position of the steady arm support.

The tension $T$ of the contact and messenger wires is imposed in the first span. The equation associated to this constraint can be written as

$$
\mathrm{f}_{\mathrm{c} 1}^{e}{ }^{2}+\mathrm{f}_{\mathrm{c} 2}^{e}{ }^{2}+\mathrm{f}_{\mathrm{c} 3}^{e}{ }^{2}-T^{2}=0
$$

where $\mathrm{f}_{\mathrm{c} k}^{e}$ is the $k$ component of the nodal force vector of the element. The groups of elements used to achieve this equation are the elements between the first mast 
and the first dropper in the messenger wire and the first steady arm and the first dropper in the contact wire.

To sum up, the steady state configuration is found by solving a system of nonlinear equations (equation 9) under constraints (equations 10 and 11). This problem can be solved using the Lagrange multiplier and Newton-Raphson methods.

\subsection{Numerical integration procedure}

To perform the time integration, the PACDIN code uses the same non-linear model of the catenary obtained from the initial configuration problem. As the undeformed length has already been obtained, the only unknown is the vector $\mathbf{q}$ in every time step. In this case, the pantograph degrees of freedom are included, as well as the contribution to the elastic $\mathbf{F}^{\mathrm{e}}$ and gravitational $\mathbf{F}^{\mathrm{g}}$ of the pantograph and the interaction element (equation 8). The following equation must be solved:

$$
\mathbf{M} \ddot{\mathbf{q}}+\mathbf{F}^{\mathrm{d}}(\dot{\mathbf{q}})+\mathbf{F}^{\mathrm{e}}(\mathbf{q})+\mathbf{F}^{\mathrm{g}}(\mathbf{q})=\mathbf{F}^{\mathrm{ext}}
$$

where $\mathbf{F}^{\mathrm{d}}$ is the vector of damping forces and $\mathbf{F}^{\text {ext }}$ the vector of external nongravitational loads. In this case, the external force vector depends only on the two parameters of the pantograph $F_{p}$ and $z_{p}$.

Once the static equilibrium has been obtained, equation 12 can be integrated to solve the dynamic problem using a non-linear time integration scheme. However, in the benchmark we use a linearized model of the catenary obtained from the initial configuration. The global stiffness matrix is obtained as the derivative of the elastic force vector $\mathbf{K}=\frac{\partial \mathbf{F}^{\mathrm{e}}(\mathbf{q})}{\partial \mathbf{q}}$. Note that this matrix includes the interaction stiffness due to the contribution of the penalty spring. The catenary damping matrix $\mathbf{C}$ is proportional to the mass and stiffness matrices, as defined in the benchmark data. The global position can be written as $\mathbf{q}=\mathbf{q}\left(t_{0}\right)+\mathbf{u}$, and the standard system of differential equations is obtained:

$$
\mathbf{M u}+\mathbf{C u}+\mathbf{K u}=\mathbf{F}^{\text {ext }}
$$

Two sources of non-linear behaviour are taken into account: the dropper slackening and the contact (change of the pantograph position and possible non-contact). The HHT method [7] is used to solve the system defined in equation 13. The time integration step for all the simulations was $\Delta t=10^{-3} \mathrm{~s}$.

\section{Additional methods available}

The PACDIN code is able to deal with different catenaries configurations,e.g. with or without stitch wires, and can include different types of spans as transitions. The user has only to choose the type of catenary and define the general geometrical data. The preprocessor then automatically generates a tentative finite element mesh, and the initial configuration module computes the static equilibrium and the undeformed length of the elements that fulfil the constraints imposed during catenary stringing. Track paths other than straight paths can be defined. For example, figure 5 shows a catenary with stitch wire in a curve.

More sophisticated pantograph models can also be used, including multiple contact strips and lateral variations of the contact point, as well as rotational degrees of freedom of the strips. PACDIN is currently being modified to include aerodynamic forces caused by wind action on catenary wires. 


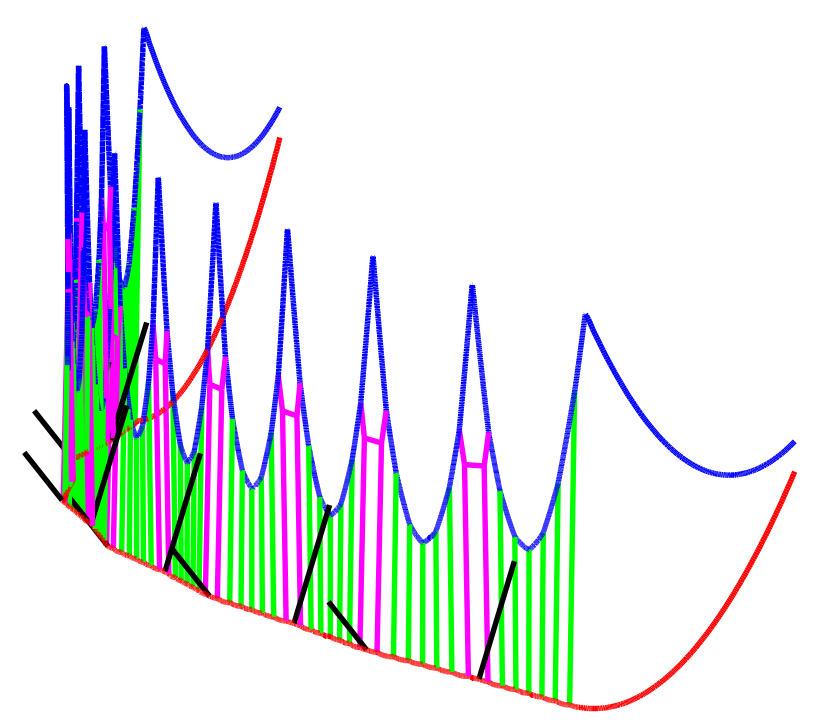

Figure 5. Catenary with stitch wire in a non-straight track.

\begin{tabular}{|l|c|c|c|c|}
\hline & \multicolumn{2}{|c|}{$250 \mathrm{~km} / \mathrm{h}$} & \multicolumn{2}{c|}{$300 \mathrm{~km} / \mathrm{h}$} \\
\hline & Reference & PACDIN & Reference & PACDIN \\
\hline 1. $F_{M}(N)$ & $110-120$ & 119.6 & $110-120$ & 118.8 \\
\hline 2. $\sigma(N)$ & $26-31$ & 28.5 & $32-40$ & 34.0 \\
\hline 3. Stat. max. $(N)$ & $190-210$ & 205.1 & $210-230$ & 220.9 \\
\hline 4. Stat. min. $(N)$ & $20-40$ & 34.1 & $-5-20$ & 16.7 \\
\hline 5. Real max. $(N)$ & $175-210$ & 178.3 & $190-225$ & 208.6 \\
\hline 6. Real min. $(N)$ & $50-75$ & 56.4 & $30-55$ & 49.0 \\
\hline \multirow{3}{*}{ 7. Uplift $(m m)$} & $48-55$ & 52.0 & & 57.5 \\
\cline { 2 - 3 } & & 49.0 & $55-65$ & 63.0 \\
\cline { 2 - 3 } & & 48.6 & & 58.9 \\
\hline
\end{tabular}

As has been pointed out above, the code is based essentially on a non-linear finite element formulation. The time integration of the equations of motion can be performed with the complete non-linear model, including large deformations, aerodynamic forces, etc. The user can choose between three integration schemes: HHT, Newmark or 4th order Runge-Kutta.

\section{Validation of the software}

Standard EN50318 [8] can be used to validate a simulation software for the dynamic interaction between pantographs and the overhead system. One part of the validation consists of performing a simulation using a reference problem with a proposed catenary and pantograph. The PACDIN code was used to do this and the results were within the range of values admitted by the standard to validate the simulation code as shown in table 2 . 


\section{Considerations on the results}

The method used in the PACDIN code to obtain the catenary steady state configuration was outlined in Section 2.4. This method makes it possible to completely fulfill all the geometric constraints imposed by the benchmark data, i.e. the height of the contact wire in certain positions and the $x$ and $y$-coordinates of droppers and steady arms connection points, all of which is performed with a non-linear large deformation finite element model. The computational cost of the method is negligible when compared to that of dynamic simulation.

The same non-linear finite element model of the catenary is linearized and then used to perform the time integration of the equations. The value of the average force in every simulation is prescribed by the benchmark data. In all cases these values are fulfilled with a precision of $\pm 0.1 N$. In general terms, the results obtained from the PACDIN code are in good agreement with the mean value of all the results compared in the benchmark. The results with higher discrepancy from the mean value are those of the rms of contact force for a speed of $V=320 \mathrm{~km} / \mathrm{h}$ in the range of frequencies between $5-20 \mathrm{~Hz}$. This discrepancy is not reproduced for the other speeds. Unlike the $3 \mathrm{D}$ case, in $2 \mathrm{D}$ case the $y$ displacement is constrained to be 0 and the steady arm is a vertical bar, thus the dynamic behavior of the structure can be affected. The agreement with the benchmark results is, in general, better in the $3 \mathrm{D}$ catenary model simulations.

\section{Conclusions}

The PACDIN code is based on a non-linear large deformation finite element catenary model. The main feature of the code is that the same finite element model is used to solve the steady state configuration and the dynamic analysis (with or without model linearization). A general formulation is used to solve the initial catenary configuration that can be used to deal with many different catenary types, with or without stitch wire, transitions, non-straight track paths, etc., without the need to modify the code, which means it is suitable for adding new configurations.

The non-linear finite element model can be used to perform non-linear simulations of the wind effect on catenary deformation, a part of the code which is currently under development.

The results of the PACDIN code are found to be in good agreement with the average value of all the codes participating in the benchmark.

\section{Acknowledgement}

The authors wish to thank Generatitat Valenciana for the financial support received in the framework of the PROMETEO 2012/023 Programme.

\section{References}

[1] A. Shabana, Computer implementation of the absolute nodal coordinate formulation for flexible multibody dynamics, Nonlinear Dynamics 16 (1998), pp. 293-306.

[2] M. Berzeri and A. Shabana, Development of simple models for the elastic forces in the absolute nodal co ordinate formulation, Journal of Sound and Vibration 235 (2000), pp. 539-565.

[3] J. Gerstmayr and A. Shabana, Analysis of Thin Beams and Cables Using the Absolute Nodal Coordinate Formulation, Nonlinear Dynamics 45 (2006), pp. 109-130.

[4] M. Tur, L. Baeza, F. Fuenmayor, and E. Garcia, A 3D absolute nodal coordinate finite element model to compute the initial configuration of a railway catenary, Engineering Structures 71 (2014), pp. $234-243$.

[5] P. Wriggers Computational Contact Mechanics, John Wiley \& Sons, Ltd., 2002. 
6] A. Collina and S. Bruni, Numerical Simulation of Pantograph-Overhead Equipment Interaction, Vehicle System Dynamics 38 (2002), pp. 261-291.

[7] T. Hughes The Finite Element Method: Linear Static and Dynamic Finite Element Analysis, Prentice Hall, New Jersey, 1987.

[8] EN50318:2002 Railway applications. Current collection systems. Validation of simulation of the dynamic interaction between pantographs and overhead contact, 2002. 